\title{
Reduction of electromagnetic exposure using hybrid (DVB-H/UMTS) networks
}

\author{
M. Schack, P. Unger, and T. Kürner \\ Institute for Communications Technology, Braunschweig Technical University, Germany
}

\begin{abstract}
The hybrid mobile communication network described in this paper consists of a point-to-point network (UMTS) and a point-to-multipoint network (DVB-H). Using an additional DVB-H network increases the downlink capacity of the communications system. Another benefit of combining these two networks is an optimised transfer of data by collecting several user requests for a single response via the broadcast network DVB-H. It is analysed how the hybrid network structure influences the electromagnetic exposure. Therefore, realistic scenarios have been developed consisting of different user behaviour and different network structures. These scenarios provide building data for investigations of indoor coverage and realistic propagation of signals. In order to evaluate the grade of exposure, criteria have been defined. These criteria have been used for comparing a hybrid network with a single UMTS network in terms of electromagnetic exposure. The simulation results of the scenarios are shown for different network structures and network configurations.
\end{abstract}

\section{Introduction}

In the last years, the reception of multimedia services in mobile environments has become more and more important. Multimedia-based content can be shown on the mobile terminals in higher qualities due to the increasing computing power and improved display technologies. This trend will surely lead to an increasing demand of such mobile services in the coming years. Many of these services will be asymmetric and interactive, whereas users receive large amounts of data. The transmission of an increasing amount of data would overload today's communication networks like UMTS (Universal Mobile Telecommunications System) which offers high data rates up to $384 \mathrm{kbit} / \mathrm{s}$. Especially the transmission of the same data which is consumed by many users at the same time and at a similar place would allocate a significant

Correspondence to: M. Schack

(schack@ifn.ing.tu-bs.de) amount of resources. Broadcast systems would then be more suitable to serve these users. Broadcast networks provide broadband access serving many users simultaneously but do not support interactivity.

The introduction of a hybrid network, the combination of a unicast (bidirectional point-to-point) and a broadcast (unidirectional point-to-multipoint) network, could provide both broadband access to many users and individual interactive channels. The benefits of both systems will be combined in order to optimise the transfer of data. On the one hand, bidirectional point-to-point channels are used for individual services. On the other hand, a unidirectional broadcast channel offers high data rates at the downlink. Requests for the same content at the same time can be combined to serve all users by only one transmission instead of individual transmissions. In this way capacity can be saved and UMTS cells can be unloaded.

The hybrid network deployed here combines the mobile communication network UMTS (Universal Mobile Telecommunications System) and the broadcast system DVB-H (Digital Video Broadcasting - Handheld). DVB-H is a broadcast system based on DVB-T (Digital Video Broadcasting Terrestrial). The DVB-H system is optimised for small devices such as mobile phones by using time slicing for less power consumption and an additional forward error correction (FEC) providing more robust signals in mobile environments (Reimers, 2005).

Since the electromagnetic exposure of radio communication systems is seen critical by the public, the reduction of the exposure is an important issue. The goal of this paper is to compare a hybrid network with a single UMTS network in terms of electromagnetic exposure. Since both network types have to serve, for comparison, the same amount of user requests, the capacity of a single UMTS network has to be increased by additional base stations (Node-Bs). A benefit of the hybrid network is expected due to the broadcast capability of the DVB-H network. Especially at hot-spot areas, where many users are concentrated, requesting the same content at the same time, one DVB-H transmitter could replace

Published by Copernicus Publications on behalf of the URSI Landesausschuss in der Bundesrepublik Deutschland e.V. 


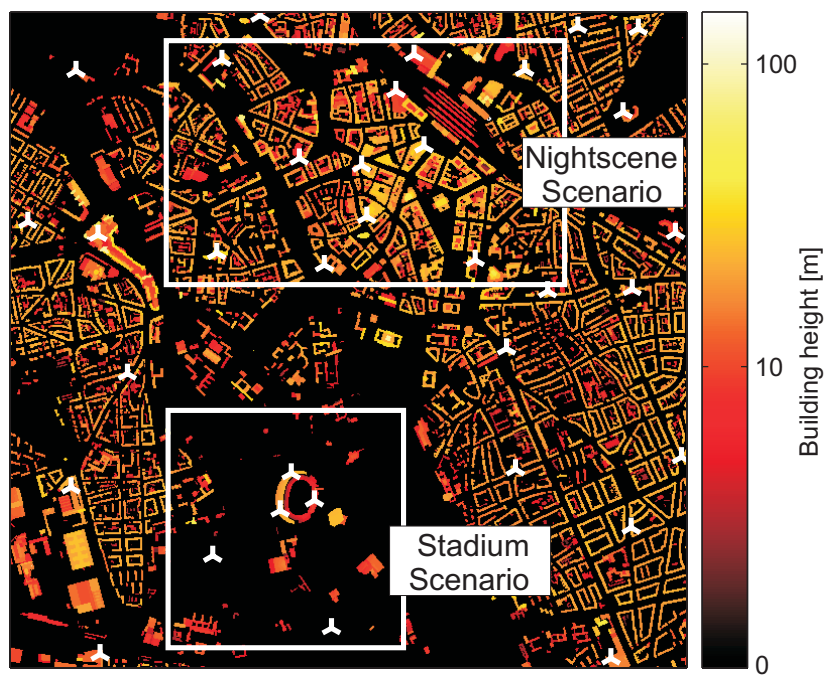

Fig. 1. Areas and building data of the scenarios Stadium and Nightscene and the planned UMTS reference network.

lots of Node-Bs. In this case, small cell structures are assumed for the DVB-H network covering the hot-spot areas. For the DVB-H network, different system configurations, i.e. different network structures and modulation schemes, are applied in order to evaluate the amount of electromagnetic exposure.

The following sections of the paper are organised as follows. The scenarios and offered services are described in detail in Sect. 2. In Sect. 3, the estimation of the power of the UMTS reference network is shown. Section 4 describes the two possibilities to enhance the UMTS network. Section 5 shows a theoretical analysis of the electromagnetic exposure and an evaluation of the simulation results. Finally, the conclusions of the study are summarised in Sect. 6 .

\section{Scenarios and services}

For the comparison of the both network types, two scenarios have been developed under consideration of different assumptions on user behaviour and geographical user distribution. The areas of the selected scenarios are shown in Fig. 1. On the one hand, a hot-spot area has been defined at the fully occupied football stadium (about 50000 spectators) of Hanover, Germany. At the Stadium scenario, replays of football highlights can be requested in the form of video streams. On the other hand, a so-called Nightscene scenario has been developed in which live information of locations is offered to visitors of bars and restaurants.

For the investigations two different service types have been defined. On the one hand, a service mix according to Ferreira et al. (2003) hab been defined, which includes e.g. voice and video telephony and which is expected to be transmitted exclusively by the UMTS network. On the other hand, a hybrid service has been defined that can be transmitted by the UMTS network or the DVB-H network. The hybrid service is assumed to consist of video streams with a data rate of $384 \mathrm{kbit} / \mathrm{s}$.

\section{Power estimation of the UMTS reference network}

For the entire city of Hanover, a UMTS reference network consisting of macro cells has been designed to fulfil the user requests for the eight typical UMTS services. The network design process is based on a snapshot simulation where users are randomly generated at the scenario area. This process is described in detail in Unger et al. (2006a). The resulting UMTS reference network structure consisting of 34 Node-Bs at roof tops for the city of Hannover in an area of $3.4 \mathrm{~km} \times 3.3 \mathrm{~km}$ is shown in Fig. 1. For each Node-B the same sectorisation, tilt and antenna type is applied. For the estimation of the utilisation of a UMTS network and the calculation of the transmitting power, the DL load according to Holma and Toskala (2005) is used. If a certain threshold for the DL load is reached, no further users can be assigned to the cell. In this case the cell is working at full capacity. The threshold for the downlink load factor has been set to 0.7 according to Laiho et al. (2002).

A typical value for the maximum nominal power of the amplifier of macro cells is $43 \mathrm{dBm}$ (Holma and Toskala, 2005). This maximal available value is used for the control channels as well as for the dedicated traffic channels. The part for the control channels is approximately 10-20 percent of the total power. In many cases the maximum transmitting power is not reached at all. In order to estimate the electromagnetic exposure, the actual transmitting power and not the maximum power is used in this investigation. Thus, the transmitting power can be tuned accurately with the concrete user behaviour. The actually resulting transmitting power of the base station $P_{\text {res }}$ arises as a result of the addition of the control channel power $P_{\text {control }}$ and the user-dependent power $P_{\text {user }}$.

As considered in this paper, for the UMTS network the same pilot power (P-CPICH) of $33 \mathrm{dBm}$ for all Node-Bs is used. Changing the pilot may cause problems during the planning process and particularly in operating UMTS networks, e.g. due to the decrease of the soft handover regions between cells. All further control channels are estimated to $30 \mathrm{dBm}$, thus the resulting total power of all control channels $P_{\text {control }}$ is $34.76 \mathrm{dBm}$. This power is independent on the utilisation of the base station and is permanently present. The power required for the connections of the individual users depends on the number and distance of the users and on the used services. The computation of the user-dependent power $P_{\text {user }}$ is described in Laiho et al. (2002). 


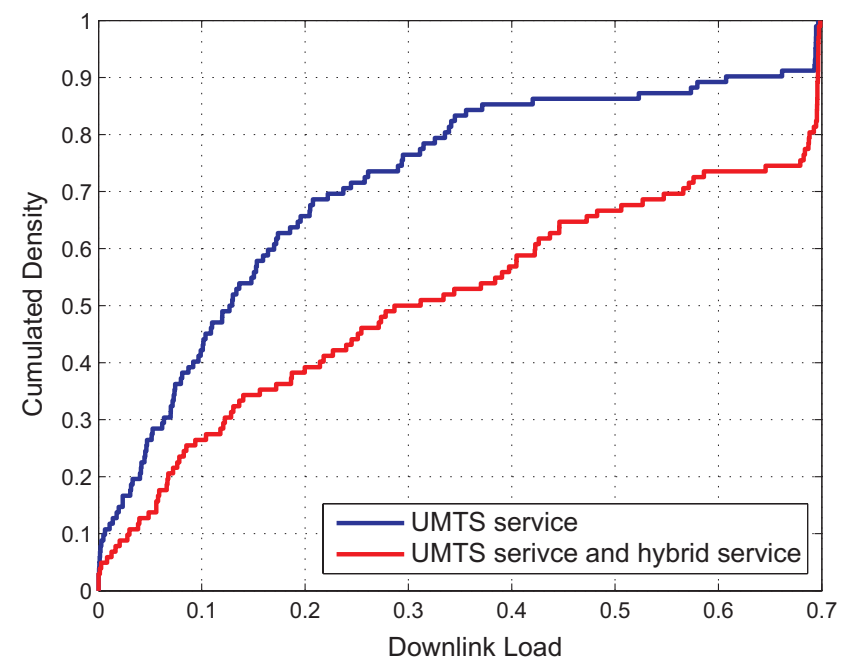

Fig. 2. Distribution of the downlink load factor of the UMTS reference network including the hybrid service of the Stadium scenario.

\section{Enhancing the UMTS network}

If the hybrid service is offered to the scenarios besides the eight UMTS services, an overloading of the UMTS network is expected. Due to the high downlink data rate of the hybrid service, the downlink load increases significantly. Figure 2 shows the cumulative density function of the downlink load for the UMTS reference network. On the one hand, the typical UMTS services are considered and on the other hand, additionally, the hybrid service of the Stadium scenario is included. It is shown that the probability of a load factor above 0.6 increases from $11 \%$ to $26 \%$ if the hybrid service is considered. The number of Node-Bs with a downlink load close to the threshold of 0.7 is increased almost by double, which leads to a rejection of users.

A large number of users could not be assigned to a NodeB. The detailed rejection rates of the services of both scenarios are given in Unger et al. (2006a). Since this heavy traffic mix cannot be handled by the UMTS reference network, it has to be enhanced in order to serve all user requests. One possibility is to enhance the capacity of the UMTS reference network by additional Node-Bs. Other enhancements of UMTS such as HSDPA (High Speed Downlink Packet Access) and MBMS (Multimedia Broadcast/Multicast Service) are not considered in this investigation. It is assumed that these concepts are not suitable for these scenarios. HSDPA is designed for individual services only (Holma and Toskala, 2006). MBMS does not offer sufficient data rate (up to $256 \mathrm{kbit} / \mathrm{s}$ specified) in order to transmit the considered services (3GPP, 2003).

Figure 3 shows the cumulative density function of the downlink load for the UMTS reference network if the hybrid service for the Nightscene service will be included. The

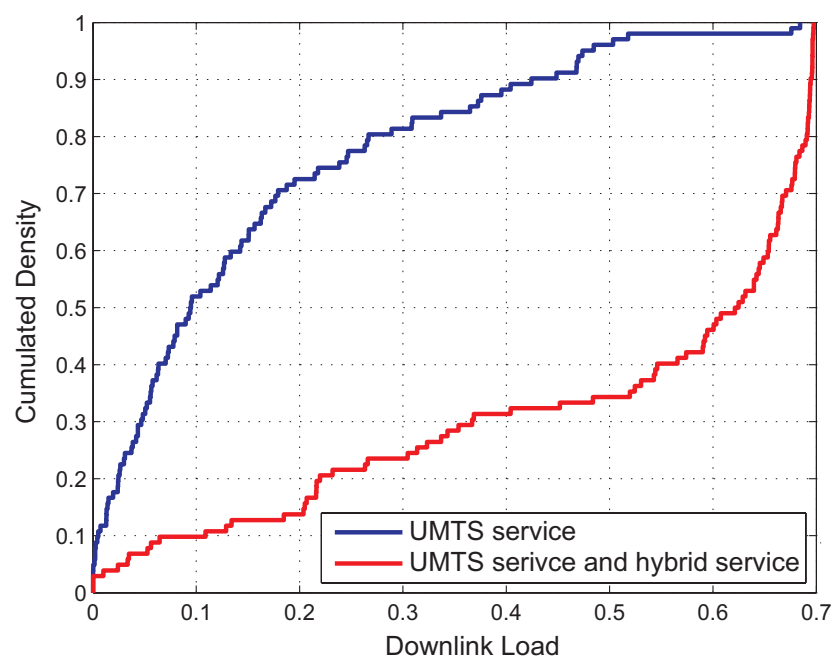

Fig. 3. Distribution of the downlink load factor of the UMTS reference network including the hybrid service of the Nightscene scenario.

percentage of Node-Bs with a load factor above 0.6 increases from $2 \%$ to $52 \%$. This is caused by the wide distribution of hot spots. Therefore, more Node-Bs are used for transmitting the hybrid service. At the simulated snapshots, about 116 of 267 users requesting the hybrid service and 37 of 521 users requesting UMTS services are rejected on average.

Another possibility to enhance the UMTS reference network is an additional DVB-H network. It can be applied in order to form a hybrid network and thus to extend the downlink capacity by a high data rate broadcast channel. It is assumed that all requests for the hybrid service are served by the DVB-H network due to the highly loaded UMTS cells. At the Stadium scenario, one DVB-H-transmitter covering a cell with a radius of $400 \mathrm{~m}$ is placed at the north side of the stadium with an antenna height of $30 \mathrm{~m}$. At the stadium scenario, one DVB-H transmitter with an antenna height of $90 \mathrm{~m}$ deploying a cell with a radius of $100 \mathrm{~m}$ has been used. For each scenario, omnidirectional antenna characteristics are assumed for the transmitter. For both scenarios, the estimated transmitting power of DVB-H, based on the specified coverage area and the link budget, is shown in Table 1. With an increasing data rate, the necessary transmitting power increases due to higher carrier-to-noise ratio values based on ETSI (2005).

\section{Exposure evaluation}

In this section, the enhanced UMTS network and the hybrid network, composed of the UMTS reference network and the DVB-H network, will be compared in terms of the arising electromagnetic exposure. In Eq. (1) the exposure ratio U 
Table 1. Transmitting Power for different modulation schemes for the DVB-H 8K mode with Guard Interval 1/4 and MPE-FEC 3/4.

\begin{tabular}{lllll}
\hline \multicolumn{1}{c}{ DVB-H } & \multicolumn{3}{c}{ EIRP [dBm] } \\
\hline $\begin{array}{l}\text { Modulation } \\
\text { scheme and code } \\
\text { rate }\end{array}$ & $\begin{array}{l}\text { Data rate } \\
{[\mathrm{Mbit} / \mathrm{s}]}\end{array}$ & $\begin{array}{l}\text { CNR } \\
{[\mathrm{dB}]}\end{array}$ & Stadium & Nightscene \\
\hline $\begin{array}{l}\text { QPSK; CR=1/2 } \\
\text { QPSK; CR=2/3 }\end{array}$ & 3.74 & 9.6 & 35.0 & 57.1 \\
$\begin{array}{l}\text { 16-QAM; } \\
\text { CR=1/2 }\end{array}$ & 7.46 & 12.6 & 38.0 & 60.1 \\
$\begin{array}{l}\text { 16-QAM; } \\
\text { CR=2/3 }\end{array}$ & 9.95 & 15.1 & 40.5 & 62.6 \\
\hline
\end{tabular}

according to Unger et al. (2006b) is defined.

$U=\left(\frac{E_{2140 \mathrm{MHz}}}{E_{L, 2140 \mathrm{MHz}}}\right)^{2}+\left(\frac{E_{500 \mathrm{MHz}}}{E_{L, 500 \mathrm{MHz}}}\right)^{2}$

The field strength value of the network is related to the reference level for the electromagnetic exposure at the appropriate frequency. These reference levels are based on the general public exposure values in ICNIRP Guidelines (1998) and may not be exceeded. The reference level $E_{L, 500 \mathrm{MHz}}$ is $30.7 \mathrm{~V} / \mathrm{m}$ for the DVB-H network and $E_{L, 2140 \mathrm{MHz}}$ equals $61 \mathrm{~V} / \mathrm{m}$ for the UMTS network. This formula can be used to compare the different network types for each point of the selected area. For a statistical evaluation of the exposure, the mean value $\bar{U}$, the maximum value $\widehat{U}$ and the $95 \%$-quantile $\tilde{U}$ are calculated.

\subsection{Theoretical analysis}

Before realistic scenarios will be simulated and evaluated, a theoretical analysis has been carried out to show how the transmitter position and the selected area influence the simulation results. The idealised scenario consists of an area with a size of $2 \mathrm{~km} \times 2 \mathrm{~km}$ with transmitters sending at a frequency of $2140 \mathrm{MHz}$. One transmitter with an antenna height of $40 \mathrm{~m}$ with a transmitting power (EIRP) of $61 \mathrm{dBm}$ is placed in the middle of the area. In order to estimate the propagation loss, the COST-231 Hata model (COST, 1999) for suburban areas has been used. The COST-231 Hata model has been designed for the frequency range from $500 \mathrm{MHz}$ to $2000 \mathrm{MHz}$, but it is expected that this model is also valid for $2140 \mathrm{MHz}$. Equation (2) specifies the calculation of the path loss $L$ depending on the frequency $f$, the height of the transmitter $H_{B S}$ and the distance from the transmitter to the mobile station $d$ for a fixed antenna height of the mobile station between 1 and $2 \mathrm{~m}$.

$L=46.3+33.9 \cdot \log f[\mathrm{MHz}]-13.8 \cdot \log H_{B S}[\mathrm{~m}]$

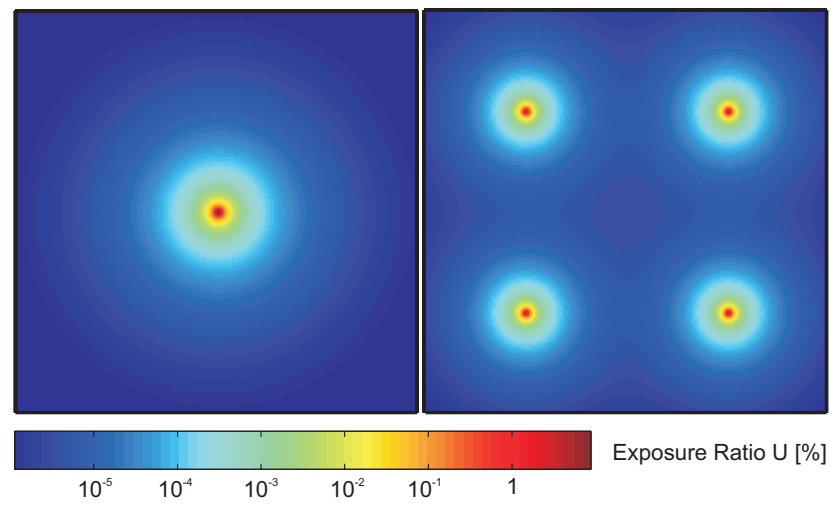

Fig. 4. Evaluation of the exposure ratio depending on the transmitter location.

Table 2. Exposure ratio values depending on the transmitter position.

\begin{tabular}{lcc}
\hline Exposure ratio [\%] & Single transmitter & 4 transmitters \\
\hline Maximum value $\widehat{U}$ & 8.74 & 2.185 \\
Mean value $\bar{U}$ & $1.7 \cdot 10^{-3}$ & $1.7 \cdot 10^{-3}$ \\
95\%-quantile $\tilde{U}$ & $4.37 \cdot 10^{-6}$ & $3.29 \cdot 10^{-6}$ \\
\hline
\end{tabular}

$+\left(44.9-6.55 \cdot \log H_{B S}[\mathrm{~m}]\right) \cdot \log (d)[\mathrm{km}]$

It was investigated how the network structure influences the exposure ratio values. One transmitter is compared to 4 transmitters which have the same accumulated transmitting power of $61 \mathrm{dBm}$. Figure 4 shows the exposure ratio values of both scenarios. It is obvious that the maximum value is caused by the single transmitter. Table 2 summarises the exposure ratio values. The four-transmitter scenario with a distributed transmitting power is beneficial in terms of the maximum value and $95 \%$-quantile of the exposure ratio values.

The statistic evaluation of the mean value and 95\%quantile are based on the considered area. Thus, the size of the selected area is important for the resulting statistic values. The closer the selected area is to the transmitter, the larger the values for mean value and $95 \%$-quantile will occur. In Table 3 the resulting values for the single-transmitter scenario with different sizes of the area are shown. The maximum value remains constant since the transmitter is placed in the middle of the selected areas. Instead, the mean value and $95 \%$-quantile change significantly. In order to evaluate the exposure ratio for different network types, the selected area has to be of the same size. Furthermore, the maximum value has to be included since it has a significant influence on the mean value and $95 \%$-quantile. 
Table 3. Exposure ratio values depending on the size of the selected area.

\begin{tabular}{llll}
\hline Exposure ratio [\%] & $4 \mathrm{~km}^{2}$ & $1 \mathrm{~km}^{2}$ & $\frac{1}{4} \mathrm{~km}^{2}$ \\
\hline Maximum value $\widehat{U}$ & 8.74 & 8.74 & 8.74 \\
Mean value $\bar{U}$ & $0.17 \cdot 10^{-2}$ & $0.67 \cdot 10^{-2}$ & $2.6 \cdot 10^{-2}$ \\
$95 \%$-quantile $\tilde{U}$ & $4.37 \cdot 10^{-6}$ & $1.31 \cdot 10^{-5}$ & $1.45 \cdot 10^{-4}$ \\
\hline
\end{tabular}

\subsection{Simulation results of realistic scenarios}

In order to evaluate the exposure for the realistic scenarios, simulations have been performed. Therefore, propagation calculations have been carried out for each transmitter using a propagation model for dense urban areas (Kürner and Meier, 2002) based i.a. on building data. Figure 5 depicts the exposure ratio values of the Stadium scenario for the mean value $\bar{U}$ and the $95 \%$-quantile $\tilde{U}$ depending on the data rate of the DVB-H network. The exposure ratio values $\bar{U}_{\text {hybrid }}$ and $\tilde{U}_{\text {hybrid }}$ for the hybrid network increase with an increasing data rate due to the higher transmitting power of the DVB-H transmitter. The maximum value $\hat{U}$ is not depicted in this figure, since it is solely caused by the UMTS network in both network types. Therefore, the maximum value of the hybrid network is not dependent on the modulation scheme of the DVB-H network. Maximum exposure ratio values of $\hat{U}_{\text {UMTS }}=0.83 \%$ and $\hat{U}_{\text {hybrid }}=0.61 \%$ occur for the enhanced UMTS network and for the hybrid network, respectively. The maximum exposure ratio value is reduced because the transmitting power of the Node-Bs at the hot spot can be reduced while serving users by the DVB-H network. Thereby, the reference level will not be exceeded by both network types. Furthermore, the exposure ratio values of the hybrid network regarding all criteria are lower than the values caused by the UMTS network for all modulation schemes of DVB-H.

Figure 6 shows the exposure ratio values for the Nightscene scenario in log scale. The results for the hybrid network show that the exposure ratio values increase for higher data rates. The maximum value of the exposure ratio also depends on the data rate of the DVB-H network. This can be explained by the fact that this value is caused by the single DVB-H transmitter due to its high transmitting power. For the highest data rate, the maximum value for the hybrid network is larger than the value for the enhanced UMTS network. Furthermore, the exposure values for mean value and 95\%-quantile of the hybrid network are higher than the values of the UMTS network.

\section{Conclusions}

The reduction of the electromagnetic exposure of radio systems is an important issue. The accuracy of the prediction of the arising electromagnetic exposure depends considerably

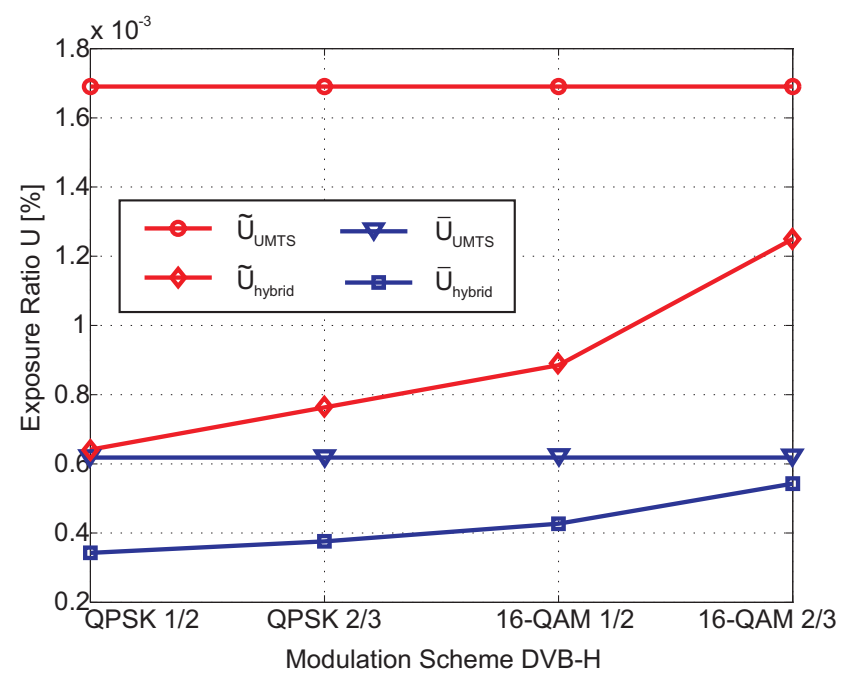

Fig. 5. Exposure ratio for both systems depending on the DVB-H modulation scheme at the Stadium scenario.

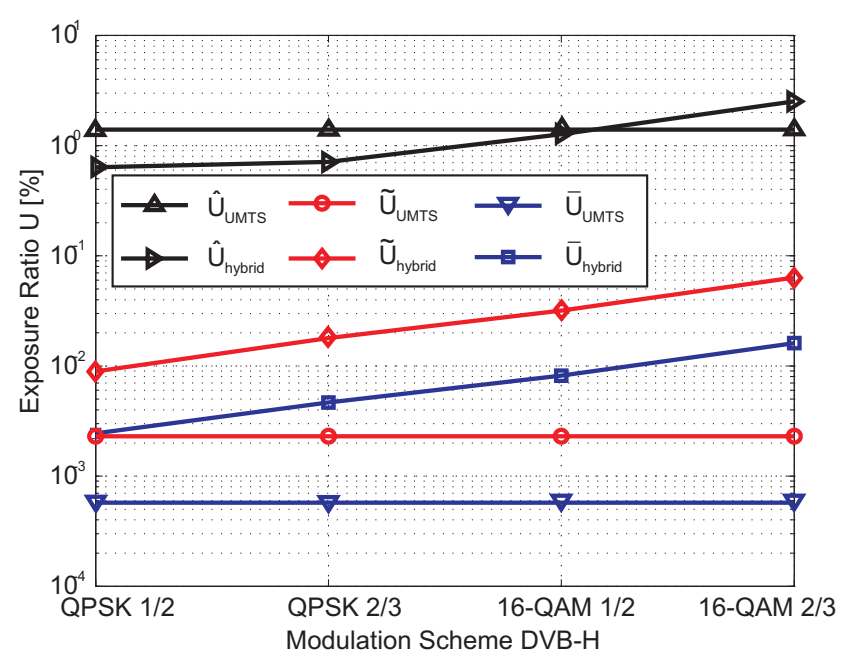

Fig. 6. Exposure ratio for both systems depending on the DVB-H modulation scheme at the Nightscene scenario.

on the accurate estimation of the transmitting power. On the one hand, the transmitting power of the UMTS Node-Bs is not determined by the maximum value, but by the estimation of the actually resulting transmitting power. On the other hand, the transmitting power of DVB-H is based on the specified coverage area and the DVB-H link budget. The results show that especially at hot-spot scenarios hybrid networks are able to minimise the electromagnetic exposure, because less Node-Bs are necessary to serve the same amount of user requests. On the other side, hybrid networks show limits in large area scenarios, where the hot spots are widely distributed.

Acknowledgements. This work has been funded by the Federal Ministry of Education and Research (Bundesministerium für Bildung und Forschung, BMBF) within the project miniWatt II. 


\section{References}

COST Action 231: Digital mobile radio towards future generation systems, final report, tech. rep., European Communities, EUR 18957, 1999.

Digital Video Broadcasting (DVB): DVB-H Implementation Guidelines, ETSI Std. TR 102377 V1.2.1, November 2005.

Ferreira, L., Correia, L. M., Xavier, D., Vasconcelos, I., and Fledderus, E. R.: Final report on traffic estimation and services characterisation, IST-2000-28088 MOMENTUM, Tech. Rep. D1.4, 2003.

Holma, H. and Toskala, A.: WCDMA for UMTS - Radio Access For Third Generation Mobile Communications, 3rd ed. John Wiley \& Sons, 2005.

Holma, H. and Toskala, A.: HSDPA/HSUPA for UMTS - High Speed Radio Access for Mobile Communications, 1rd ed. John Wiley \& Sons, 2006.

ICNIRP Guidelines: Guidelines for limiting exposure to timevarying electric, magnetic and electromagnetic fields (up to 300 GHz), Health Physics, 74(4), 494-522, 1998.
Kürner, T. and Meier, A.: Prediction of Outdoor and Outdoor-toIndoor Coverage in Urban Areas at $1.8 \mathrm{GHz}$, IEEE Journal on Selected Areas in Communications, 20(3), 496-506, April, 2002.

Laiho, J., Wacker, A., and Novosad, T.: Radio network planning and optimisation for UMTS, John Wiley \& Sons, 1st ed., 2002.

Reimers, U. (Ed.): DVB - The Family of International Standards for Digital Video Broadcasting, 2nd ed. Springer: Berlin, Heidelberg, New York, 2005.

Unger, P., Schack, M., and Kürner, T.: Impact of the Hybrid (DVBH/UMTS) Network Structure on the Electromagnetic Exposure, International Symposium on Wireless Communication Systems - ISWCS, Valencia, 5-8 September, 2006a.

Unger, P., Schack, M., and Kürner, T.: Minimising the Electromagnetic Exposure at Hot-Spot Areas using Hybrid (DVB-H/UMTS) Networks, German Microwave Conference GeMIC 2006, Karlsruhe, 28-30 March, 2006b.

3GPP TS 25.346: Technical Specification Group Radio Access Network; Introduction of the Multimedia Broadcast Multicast Service (MBMS) in the Radio Access Network (Stage-2), March, 2003. 